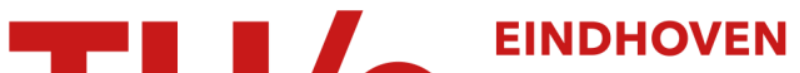 \\ UNIVERSITY OF \\ TECHNOLOGY
}

\section{Job shop scheduling by local search}

Citation for published version (APA):

Aarts, E. H. L., Lenstra, J. K., Laarhoven, van, P. J. M., \& Ulder, N. L. J. (1992). Job shop scheduling by local search. (Memorandum COSOR; Vol. 9229). Technische Universiteit Eindhoven.

Document status and date:

Published: 01/01/1992

\section{Document Version:}

Publisher's PDF, also known as Version of Record (includes final page, issue and volume numbers)

\section{Please check the document version of this publication:}

-A submitted manuscript is the version of the article upon submission and before peer-review. There can be important differences between the submitted version and the official published version of record. People interested in the research are advised to contact the author for the final version of the publication, or visit the $\mathrm{DOI}$ to the publisher's website.

- The final author version and the galley proof are versions of the publication after peer review.

- The final published version features the final layout of the paper including the volume, issue and page numbers.

Link to publication

\section{General rights}

Copyright and moral rights for the publications made accessible in the public portal are retained by the authors and/or other copyright owners and it is a condition of accessing publications that users recognise and abide by the legal requirements associated with these rights.

- Users may download and print one copy of any publication from the public portal for the purpose of private study or research.

- You may not further distribute the material or use it for any profit-making activity or commercial gain

- You may freely distribute the URL identifying the publication in the public portal.

If the publication is distributed under the terms of Article 25fa of the Dutch Copyright Act, indicated by the "Taverne" license above, please follow below link for the End User Agreement:

www.tue.nl/taverne

Take down policy

If you believe that this document breaches copyright please contact us at:

openaccess@tue.nl

providing details and we will investigate your claim. 
EINDHOVEN UNIVERSITY OF TECHNOLOGY

Department of Mathematics and Computing Science

Memorandum COSOR 92-29

Job Shop Scheduling by

Local Search

E.H.L. Aarts

P.J.M. van Laarhoven

J.K. Lenstra

N.L.J. Ulder

Eindhoven, July 1992

The Netherlands 
Eindhoven University of Teclinology

Department of Mathematics and Computing Science

Probability theory, statistics, operations research and systems theory P.O. Box 513

5600 MB Eindhoven - The Netherlands

Secretariate: Dommelbuilding 0.0:3

Telephone: $\quad 010-473130$

ISSN 09264493 


\title{
Job Shop Scheduling by Local Search
}

\author{
E.H.L. Aarts ${ }^{1,2}$, P.J.M. van Laarhoven ${ }^{3}$, J.K. Lenstra ${ }^{2,4}$ and N.L.J. Ulder ${ }^{5}$ \\ 1 Philips Research Laboratories, P.O. Box 80000, NL-5600 JA Eindhoven, The Netherlands \\ 2 Eindhoven University of Technology, Box 513, NL-5600 MB Eindhoven, The Netherlands \\ ${ }^{3}$ McKinsey \& Company, Amstel 344, NL-1017 AS Amsterdam, The Netherlands \\ 4 CWI, P.O. Box 4079, NL-1009 AB Amsterdam, The Netherlands \\ ${ }^{5}$ Océ-Nederland B.V., P.O. Box 101, NL-5900 MA Venlo, The Netherlands
}

\begin{abstract}
We present a computational performance analysis of local search algorithms for job shop scleduling. The algorithms under investigation are iterative improvement, simulated annealing, threshold accepting and genetic local search. Our study shows that simulated annealing performs best in the sense that it finds better solutions than the other algorithms within the same amount of running time. Compared to more tailored algorithms, simulated annealing still finds the best results but only under the assumption that running time is of no concern.
\end{abstract}

Key words: Local search, iterative improvement, threshold accepting, genetic algorithms, simulated annealing, job shop scheduling.

\section{Introduction}

Combinatorial optimization problems arise in such diverse areas as computer and VLSI design, facilities layout, production scheduling, and distribution planning. Many of these problems have been proved NP-hard [Garey \& Johnson, 1979], and it is consequently believed that they cannot be solved in polynomial time. In practice this means that solving large instances of such problems to optimality requires impracticable running times. To avoid this, one often resorts to approximation algorithms that try to find near-optimal solutions within acceptable running times.

There is a second justification for the use of approximation algorithms. Many practical problems are generalizations of well-known combinatorial optimization problems, but the generalization involves complicated side constraints that cannot be readily incorporated into optimization algorithms for the underlying standard problem. Thus, even if an optimization algorithm were available for the underlying problem, it might not be applicable to the problem at hand.

From an application point of view, approximation algorithms range from tailored algorithms, designed for a specific problem type, to general algorithms, which can be applied to a broad range of problem types. For the latter, it is often more appropriate to speak of algorithmic templates since in many cases the problem specific details still need to be filled in to obtain an operational algorithm. Local search is an example of such an algorithmic template for combinatorial optimization [Papadimitriou \& Steiglitz, 1982; Yannakakis, 1991]. Traditionally, local search stands for 
iterative improvement with the notable exception of the variable-depth search algorithms proposed by Kernighan \& Lin [Kernighan \& Lin, 1971; Lin \& Kernighan, 1973]. The introduction of simulated annealing, a randomized approach to local search [Kirkpatrick, Gelatt \& Vecchi, 1983; Cerny, 1985], and its successful application to problems in many different areas [Aarts \& Korst, 1989; Van Laarhoven \& Aarts, 1987], has broadened the scope of local search and has led to new algorithmic templates such as threshold accepting [Dueck \& Scheuer, 1988], tabu search [Glover, 1989] and to variants of genetic algorithms [Goldberg, 1989]. Also certain classes of neural networks exhibit a strong relation with local search [Baum, 1986]. For a detailed overview the reader is referred to Aarts, Korst \& Zwietering [1991].

For a number of these approaches theoretical results are available regarding the asymptotic convergence to global minima. However, only little is known about the theoretical finite-time performance of local search. Therefore, many authors have been carrying out computational studies in which the various approaches are applied to specific combinatorial optimization problems, trying to reveal their real strength, for instance in comparison with tailored optimization and approximation algorithms. For example, Johnson et al. $[1989 ; 1991]$ compared simulated annealing with several other algorithms for the traveling salesman, graph coloring, graph partitioning and number partitioning problems. The general conclusion from these studies seems to be that the new approaches are eminently suitable to be applied to large instances of problems that are hard to model and for which no satisfactory tailored algorithms are available. Evidently, this area provides ample opportunity for a general approach whose implementation requires only a modicum of sophistication.

The present paper belongs to a series of papers in which we report on the computational performance of local search algorithms when applied to specific combinatorial optimization problems; in a previous paper [Ulder et al., 1990], we presented results for the traveling salesman problem [Lawler et al., 1985]. Here, we consider the job shop scheduling problem, one of the computationally more difficult combinatorial optimization problems [French, 1982; Lawler et al., 1992]. In addition to a mutual comparison, we also compared various local search approaches with a number of job shop scheduling heuristics known from the literature.

The organization of this paper is as follows. In Section 2 we define the job shop scheduling problem and describe the various neighborhoods used by the local search algorithms under investigation. Section 3 presents the algorithms compared in this paper and Section 4 contains the numerical results. The paper ends with some conclusions and final remarks.

\section{Job shop scheduling}

The job shop scheduling problem is defined as follows. Given are $n$ jobs and $m$ machines. Each job consists of a sequence of operations, which must be executed in a given order. Each operation has to be executed on a given machine for a given period of time. A machine can perform at most one operation at a time. The problem is to find a schedule, i.e., an assignment of the operations to time intervals, such that the makespan, given by the total length of the schedule, is minimal.

To apply local search, one has to define a set of feasible solutions, a cost function and a neighborhood structure. Here, we use the formulation of Roy \& Sussmann [1964], who represent an instance of job shop scheduling by a disjunctive graph $G=(V, A, E)$, where the vertex set $V$ corresponds to the set of operations, the arc set $A$ consists of arcs connecting consecutive operations of the same 


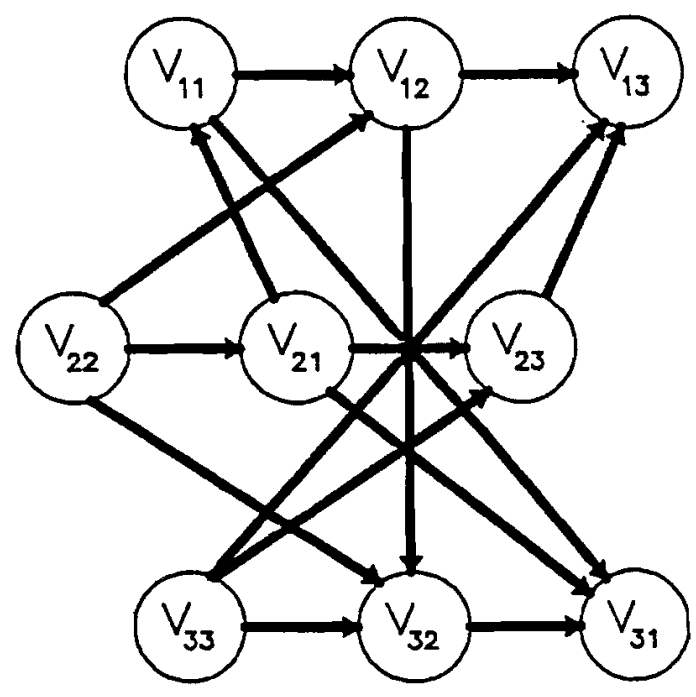

Figure 1. An example of a 3-job, 3-machine instance. Node $v_{i j}$ corresponds to an operation of job $i$ processed on machine $j$.

job, and the edge set $E$ consists of edges connecting operations that must be executed on the same machine. Figure 1 gives an example for a 3-job 3-machine instance in which each job consists of three operations. The first job is to be carried out on machines 1,2 and 3 (in that order); the second job on machines 2, 1 and 3, and the third one on machines 3,2 and 1 . Solid lines denote arcs and dotted lines denote edges. The weight of a vertex is given by the processing time of the corresponding operation. A feasible solution $i$ can be defined as a set of orientations for the edges in $E$, such that the resulting digraph $D_{i}$ is acyclic; the cost of a solution is then given by the length of the longest path in $D_{i}$. Figure 2 shows a solution for the instance of given in Figure 1. In this paper we consider the following two neighborhood structures.

$\left(N_{1}\right)$ Given a solution $i$ characterized by a digraph $D_{i}$, a neighboring solution is obtained by choosing two operations $v$ and $w$ that are immediate successors on some machine $k$ and for which the arc $(v, w)$ is on a longest path in $D_{i}$, and reversing $(v, w)$ or, in other words, reversing the order in which $v$ and $w$ are processed on machine $k$. For this neighborhood the following results are of interest [Van Laarhoven, Aarts \& Lenstra, 1992].

(i) The reversal of $(v, w)$ results in an acyclic digraph $D_{j}$, corresponding again to a feasible solution $j$.

(ii) Reversals of arcs on the longest path are the only arc reversals that can (but need not) result in a digraph with a shorter longest path than the original digraph.

(iii) For any digraph $D_{i}$, corresponding to an arbitrary solution $i$, it is possible to construct a sequence of arc reversals leading from $D_{i}$ to a digraph corresponding to a globally minimal solution. This is a necessary and sufficient condition for asymptotic convergence of simulated annealing.

$\left(N_{2}\right)$ Given a solution $i$ and its digraph $D_{i}$, a neighboring solution is obtained by chosing again two vertices $v$ and $w$ satisfying the conditions mentioned under (1), but restricting the choice 


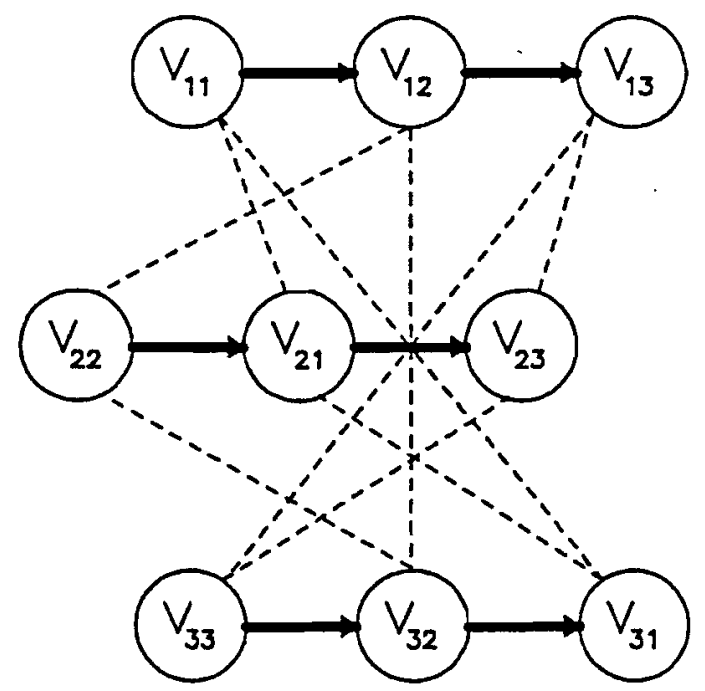

Figure 2. A solution to the instance of Figure 1.

to vertices for which at least one of the arcs $(p(v), v)$ and $(w, s(w))$ is not on a longest path, where $p(v)$ and $s(w)$ are the predecessor of $v$ and the successor of $w$ on machine $k$, respectively. This restriction is motivated by the fact that if both $(p(v), v)$ and $(w, s(w))$ are on a longest path, then the reversal of $(v, w)$ cannot lead to a shorter longest path [Matsuo, Suh \& Sullivan, 1988]. In addition to reversing $(v, w)$, also $(p(u), u)$ and $(x, s(x))$ are reversed, where $u$ is the immediate predecessor of $w$ in the job sequence of $w$ and $x$ is the immediate successor of $v$ in the job sequence of $v$. A detailed description of this neighborhood is given by Matsuo, Suh \& Sullivan [1988]. Note that in this case the neighboring solutions of a solution $i$ are obtained by reversing three arcs in $D_{i}$. Thus, this neighborhood is more intricate than the one given under (1). Finally we remark that (i) does not hold for this neighborhood since not all reversals pertain to arcs on a longest path. (ii) does not apply here and (iii) is open.

\section{The algorithms}

We now describe the algorithms used in the computational comparison. The multi-start iterative improvement and simulated annealing algorithms are only briefly mentioned; threshold accepting and genetic local search are presented in more detail.

\subsection{Multi-start iterative improvement}

Our multi-start iterative improvement algorithm consists of a number of cycles. Each cycle is started with a randomly generated solution and subsequently the neighborhood is searched for a better solution. If such a solution is found, it replaces the current one and the search continues in the neighborhood of the new solution. A cycle is terminated when a local minimum is reached, i.e. a solution whose cost is at least as good as that of all of its neighbors. After termination of a cycle 
a new one is started. This process continues until some specified limit on the running time (or a specified number of cycles) is reached. The best solution found in all cycles is returned as the final solution obtained by the algorithm.

\subsection{Simulated annealing}

Simulated annealing is a randomized version of local search. In addition to cost improving neighbors, which are always accepted, also cost deteriorating neighbors are accepted with a positive probability that gradually decreases in the course of the algorithm's execution. The lowering of the acceptance probability is controlled by a parameter, whose values are determined by a cooling schedule. Simulated annealing has been widely applied and in many cases it finds good quality solutions. For more details, the reader is referred to Aarts \& Korst [1989] or Van Laarhoven \& Aarts [1987]. A detailed study of simulated annealing for job shop scheduling is reported by Van Laarhoven, Aarts \& Lenstra [1992].

Our simulated annealing algorithm uses the cooling schedule described by Van Laarhoven \& Aarts [1987]. This is a three-parameter schedule, where the parameters $\chi_{0}$ and $\varepsilon_{\text {, determine the initial }}$ and final values of the control parameter, respectively, and the third parameter $\delta$ determines the decrement of the control parameter.

\subsection{Threshold accepting}

Threshold accepting, proposed by Dueck \& Scheuer [1988], is a deterministic version of simulated annealing. A neighbor is accepted if the difference in cost between the neighbor and the current solution is smaller than a nonnegative threshold. The threshold values vary in the course of the algorithm's execution. Initially they are large and subsequently they are gradually decreased to become zero in the end. So far, no general rules are known that determine appropriate threshold

\begin{tabular}{rcrrrrrrrrrr}
\hline$\mu$ & $\bar{f}$ & $\mu$ & \multicolumn{1}{c}{$f$} & $\mu$ & \multicolumn{1}{c}{$\bar{f}$} & $\mu$ & $\bar{f}$ & $\mu$ & $\bar{f}$ & $\mu$ & $\bar{f}$ \\
\hline 1 & 1182.2 & 6 & 1002.6 & 11 & 1011.2 & 16 & 1005.8 & 21 & 1049.6 & 26 & 1034.8 \\
2 & 1124.8 & 7 & 1007.8 & 12 & 987.4 & 17 & 1030.8 & 22 & 1032.0 & 27 & 1045.4 \\
3 & 1047.0 & 8 & 1013.8 & 13 & 1023.6 & 18 & 1010.8 & 23 & 1022.4 & 28 & 1039.4 \\
4 & 1124.8 & 9 & 1002.2 & 14 & 1012.0 & 19 & 1014.4 & 24 & 1048.0 & 29 & 1042.0 \\
5 & 1022.2 & 10 & 990.6 & 15 & 1021.8 & 20 & 1044.8 & 25 & 1022.2 & 30 & 1040.6 \\
\hline
\end{tabular}

Table 1. Average makespan $\bar{f}$ from 5 runs of threshold accepting for a 10-job, 10-machine job shop scheduling instance with 20 different sets of thresholds, each corresponding to a different value of the scaling parameter $\mu$.

values for an arbitrary optimization problem. This is in contrast to the many results on cooling schedules for simulated annealing. What is available though is the set of 30 threshold values that where successfully applied by Dueck \& Scheuer to find solutions for a 442-city instance of the traveling salesman problem. Here we used the following empirical argument to find appropriate threshold values for job shop scheduling. Table 1 contains the average values of the makespans 
obtained from five runs of threshold accepting for a 10-job 10-machine instance obtained with 30 different sets of thresholds. At each threshold value, 2000 trials are allowed. The sets of threshold values are obtained by multiplying the values of Dueck \& Scheuer by a factor $\mu \cdot \gamma$, where $\mu=1,2, \ldots, 30$ and $\gamma$ is the average difference in cost of the cost increasing trials in a random walk of length 10,000 through the set of feasible solutions. Based on the results in Table 1 and similar results obtained for other instances, we decided to use $\mu=12$ in our study. We do not rule out the possibility that fine tuning of the threshold values for job shop scheduling may yield better results.

\subsection{Genetic algorithms}

The effectiveness of multi-start iterative improvement may be improved by using the information available from the solutions obtained in the indivudual cycles. Several authors have proposed variants of local search algorithms, using ideas from population genetics; see for example, Mühlenbein, Gorges-Schleuter \& Krämer [1987], Jog, Suh \& Van Gucht [1989], and [Ulder et al., 1990]. We propose a genetic local search algorithm consisting of the following steps.

Step 1. Initialize: construct an initial population of $P$ solutions.

Step 2. Improve: use local search to replace each solution by a locally optimal one; thus, a population of $P$ local minima is obtained.

Step 3. Recombine: expand the current population by adding $P$ offspring solutions obtained by recombining $P$ pairs of solutions in the current population.

Step 4. Improve: use local search to replace each offspring solution by a locally optimal one; thus, a population of $2 P$ local minima is obtained.

Step 5. Select: reduce the extended population to its original size by selecting the best $P$ solutions.

Step 6. Iterate: repeat Steps 3 to 5 until a stopping criterion is satisfied.

We now discuss some of these steps in more detail.

Step 1. Initialization is done by randomly generating $P$ solutions.

Steps 2\&4. We use local search algorithms based on one of the neighborhood structures mentioned in Section 2.

Step 3. The candidate solutions for recombination are chosen randomly from the current population. The recombination of a pair of solutions $i$ and $j$ is based on the observation that the cost of a solution is given by the length of its longest path. Recombination is done by reversing some arcs on the longest path of $j$ such that the resulting arcs are identical to those in $i$. More specifically, we choose, at random, an $\operatorname{arc}(v, w)$ in $D_{i}$. If the arc $(w, v)$ occurs in $D_{j}$ and belongs to a longest path in $D_{j}$, it is reversed. Next the longest path in the resulting digraph $D_{j}$ is computed, and $D_{j}$ is replaced by $D_{j \prime}$. Choosing an arc in $D_{i}$ and reversing a corresponding arc in $D_{j}$ is repeated $k$ times. The net result is an offspring solution obtained from $j$ by implanting a subset of arcs from $D_{i}$ into $D_{j}$. In our implementation, $k$ is set to $\lfloor n \cdot m / 2\rfloor$. Extensive experimentation 
reveals that the precise value of $k$ is not very critical with respect to the effectiveness of the algorithms [Ulder, 1990].

Step 6. The algorithm terminates when either all solutions in the population have equal cost, or the best makespan in the population has not changed for $K$ subsequent generations (i.e., repetitions of Steps 3 to 5). In our implementation we used $K=10$.

\section{Computational results}

\subsection{Comparison of local search algorithms}

We compared the performance of the following seven algorithms: multi-start iterative improvement with neighborhoods $N_{1}$ and $N_{2}$ (MSII1 and MSII2, respectively), threshold accepting (TA), simulated annealing with $N_{1}$ and $N_{2}$ (SA1 and SA2, respectively), and genetic local search with $N_{1}$ and $N_{2}$ (GLS1 and GLS2, respectively). The comparison is carried out for a set of 43 instances. The first 40 instances are due to Lawrence [1984]; the remaining three to Fisher \& Thompson [1963]. The last three include the notorious 10-job 10-machine instance that has defied solution to optimality for more than twenty years. For all instances, the number of operations of each job equals the number of machines and each job has precisely one operation on each machine.

The algorithms were programmed in PASCAL and care was taken to have identical data structures and subroutines wherever possible. For each instance, the algorithms were allowed about equal amounts of running time; differences of at most $2 \%$ occur, with the exception of the easy D, G and $\mathrm{H}$ instances. The reference point for each instance was given by the time taken by the SAalgorithm described in Section 3.2, with the $N_{1}$ neighborhood and with the parameters values $\chi_{0}=0.95, \delta=1$, and $\varepsilon_{s}=10^{-5}$, respectively (SA1). The multi-start algorithms were terminated when their running times exceeded those of SA1. For threshold accepting we tuned the number of trials per threshold and for genetic algorithms the population size to have about the same running times as SA1; the precise values of these parameters are given in Table 2. Finally, for SA2, we used $\delta=1.15$ to equalize running times. The results of the comparison are displayed in Table 3 . The

\begin{tabular}{lrrrrrrrrrrr}
\hline & A1-A5 & B1-B5 & C1-C5 & D1-D5 & F1-F5 & G1-G5 & H1-H5 & I1-I5 & FISH06 & FISH10 & FISH20 \\
\hline$T$ & 2100 & 3600 & 5000 & 6750 & 700 & 800 & 900 & 6250 & 500 & 2100 & 1600 \\
$P_{1}$ & 55 & 45 & 33 & 33 & 25 & 30 & 25 & 60 & 45 & 35 & 20 \\
$P_{2}$ & 40 & 35 & 25 & 25 & 20 & 25 & 20 & 45 & 35 & 25 & 13 \\
\hline
\end{tabular}

Table 2. Number of trials per threshold $T$ for threshold accepting, and population sizes $P_{1}$ and $P_{2}$ for genetic local search with $N_{1}$ and $N_{2}$ neighborhoods.

entries in the table are average makespans, computed from the solutions obtained by running each algorithm five times for each instance. From Table 3 we can make the following observations.

(i) Using a more intricate neighborhood structure pays off: MSII2 finds better solutions than MSII1 and GLS2 finds better ones than GLS1. For simulated annealing, however, the difference between $\mathrm{SA} 1$ and SA2 is not significant. 


\begin{tabular}{|c|c|c|c|c|c|c|c|c|c|c|}
\hline \multirow[t]{2}{*}{ Instance } & \multirow[t]{2}{*}{$\mathbf{n}$} & \multirow[t]{2}{*}{$\mathbf{m}$} & \multicolumn{7}{|c|}{$\bar{f}$} & \multirow[t]{2}{*}{ t } \\
\hline & & & MSII1 & MSII2 & TA & SA1 & SA2 & $\overline{\text { GLS1 }}$ & GLS2 & \\
\hline A1 & 10 & 10 & 1029.6 & 1019.0 & 977.8 & 976.8 & 969.0 & 976.8 & 976.8 & 88.2 \\
\hline A2 & 10 & 10 & 858.4 & 829.2 & 829.8 & 787.2 & 785.6 & 791.2 & 791.6 & 91.4 \\
\hline A3 & 10 & 10 & 930.2 & 894.0 & 901.2 & 859.8 & 856.0 & 856.6 & 858.4 & 96.8 \\
\hline A4 & 10 & 10 & 922.6 & 899.6 & 925.8 & 855.0 & 854.6 & 863.6 & 859.0 & 93.8 \\
\hline A5 & 10 & 10 & 963.6 & 959.8 & 974.0 & 911.4 & 911.4 & 913.2 & 916.2 & 107.6 \\
\hline B1 & 15 & 10 & 1226.8 & 1181.2 & 1104.2 & 1083.6 & 1078.0 & 1084.4 & 1085.2 & 243.4 \\
\hline B2 & 15 & 10 & 1127.2 & 1099.2 & 979.6 & 950.2 & 950.2 & 954.0 & 944.0 & 254.2 \\
\hline B3 & 15 & 10 & 1164.8 & 1142.0 & 1033.7 & 1032.0 & 1032.0 & 1032.0 & 1032.0 & 242.2 \\
\hline B4 & 15 & 10 & 1111.8 & 1076.0 & 1014.6 & 962.0 & 960.4 & 970.0 & 981.2 & 234.8 \\
\hline B5 & 15 & 10 & 1167.8 & 1126.4 & 1075.4 & 1003.0 & 1019.6 & 1016.4 & 1010.0 & 254.8 \\
\hline C1 & 20 & 10 & 1473.2 & 1389.6 & 1227.0 & 1225.2 & 1221.2 & 1240.2 & 1236.2 & 469.6 \\
\hline $\mathrm{C} 2$ & 20 & 10 & 1502.4 & 1447.6 & 1289.8 & 1282.0 & 1275.6 & 1303.6 & 1300.8 & 492.0 \\
\hline C3 & 20 & 10 & 1486.4 & 1424.0 & 1286.4 & 1249.2 & 1242.8 & 1281.0 & 1264.8 & 455.6 \\
\hline C4 & 20 & 10 & 1485.2 & 1426.4 & 1262.4 & 1233.4 & 1225.8 & 1290.2 & 1260.0 & 471.0 \\
\hline C5 & 20 & 10 & 1619.2 & 1546.6 & 1366.6 & 1355.0 & 1355.0 & 1402.2 & 1386.0 & 441.4 \\
\hline D1 & 30 & 10 & 2013.0 & 1946.8 & 1784.0 & 1784.0 & 1784.0 & 1784.0 & 1784.0 & 879.8 \\
\hline D2 & 30 & 10 & 2119.4 & 2009.6 & 1850.0 & 1850.0 & 1850.0 & 1850.0 & 1850.0 & 909.0 \\
\hline D3 & 30 & 10 & 1958.4 & 1879.0 & 1719.0 & 1719.0 & 1719.0 & 1719.0 & 1719.0 & 1045.6 \\
\hline D4 & 30 & 10 & 2021.4 & 1937.8 & 1721.0 & 1721.0 & 1721.0 & 1737.0 & 1730.2 & 1055.4 \\
\hline D5 & 30 & 10 & 2158.4 & 2123.4 & 1888.0 & 1888.0 & 1888.0 & 1894.2 & 1890.2 & 1004.4 \\
\hline F1 & 10 & 5 & 670.4 & 666.0 & 682.4 & 666.0 & 666.0 & 666.0 & 666.0 & 17.2 \\
\hline F2 & 10 & 5 & 700.8 & 688.0 & 693.8 & 669.2 & 658.6 & 668.0 & 659.0 & 18.6 \\
\hline F3 & 10 & 5 & 645.0 & 633.2 & 628.2 & 617.6 & 616.6 & 613.2 & 609.2 & 21.0 \\
\hline F4 & 10 & 5 & 620.6 & 614.8 & 632.0 & 598.8 & 590.6 & 599.6 & 594.0 & 16.8 \\
\hline F5 & 10 & 5 & 593.0 & 593.0 & 593.0 & 593.0 & 593.0 & 593.0 & 593.0 & 13.6 \\
\hline G1 & 15 & 5 & 926.0 & 926.0 & 926.0 & 926.0 & 926.0 & 926.0 & 926.0 & 25.4 \\
\hline G2 & 15 & 5 & 923.0 & 915.0 & 894.0 & 890.0 & 919.8 & 890.0 & 890.0 & 42.2 \\
\hline G3 & 15 & 5 & 884.0 & 865.6 & 863.0 & 863.0 & 863.0 & 863.0 & 863.0 & 39.8 \\
\hline G4 & 15 & 5 & 952.6 & 951.0 & 951.0 & 951.0 & 951.0 & 951.0 & 951.0 & 35.6 \\
\hline G5 & 15 & 5 & 958.0 & 958.0 & 958.0 & 958.0 & 958.0 & 958.0 & 958.0 & 17.0 \\
\hline H1 & 20 & 5 & 1227.0 & 1222.4 & 1222.0 & 1222.0 & 1222.0 & 1222.0 & 1222.0 & 59.6 \\
\hline $\mathrm{H} 2$ & 20 & 5 & 1046.8 & 1039.0 & 1039.0 & 1039.0 & 1039.0 & 1039.0 & 1039.0 & 50.4 \\
\hline H3 & 20 & 5 & 1160.8 & 1151.6 & 1150.0 & 1150.0 & 1150.0 & 1150.0 & 1150.0 & 50.4 \\
\hline $\mathrm{H} 4$ & 20 & 5 & 1292.0 & 1292.0 & 1292.0 & 1292.0 & 1292.0 & 1292.0 & 1292.0 & 21.2 \\
\hline H5 & 20 & 5 & 1312.6 & 1284.4 & 1207.0 & 1207.0 & 1207.0 & 1207.0 & 1207.0 & 76.6 \\
\hline I1 & 15 & 15 & 1523.8 & 1456.6 & 1385.8 & 1307.8 & 1308.0 & 1324.8 & 1310.6 & 602.2 \\
\hline I2 & 15 & 15 & 1640.2 & 1598.8 & 1469.2 & 1440.8 & 1451.4 & 1449.4 & 1450.0 & 636.2 \\
\hline I3 & 15 & 15 & 1481.8 & 1421.8 & 1323.0 & 1235.4 & 1243.0 & 1285.2 & 1283.0 & 635.6 \\
\hline I4 & 15 & 15 & 1478.8 & 1445.4 & 1305.8 & 1258.2 & 1263.8 & 1279.0 & 1279.2 & 592.2 \\
\hline I5 & 15 & 15 & 1488.0 & 1439.0 & 1295.0 & 1256.4 & 1254.8 & 1273.2 & 1260.4 & 596.8 \\
\hline FISH06 & 6 & 6 & 56.2 & 55.0 & 60.4 & 55.0 & 55.0 & 55.0 & 55.0 & 9.4 \\
\hline FISH10 & 10 & 10 & 1055.8 & 1056.6 & 1003.8 & 969.2 & 977.8 & 978.2 & 982.2 & 99.4 \\
\hline FISH20 & 20 & 5 & 1356.0 & 1329.2 & 1228.6 & 1216.2 & 1245.0 & 1295.0 & 1294.2 & 88.4 \\
\hline
\end{tabular}

Table 3. Average makespan $\bar{f}$ and running time $\bar{t}$ for multi-start iterative improvement (MSII), threshold accepting (TA), simulated annealing (SA), and genetic local search (GLS). The additions 1 and 2 correspond to the use of neighborhood $N_{1}$ or $N_{2}$, respectively; $n$ and $m$ denote the number of jobs and machines, respectively. 
(ii) The multi-start algorithms are clearly inferior to the other algorithms. The difference becomes quite pronounced for the larger problem instances. For the D instances, for example, SA1 finds makespans that are on the average $12.8 \%$ shorter than those found by MSII1.

(iii) For those instances for which SA1 finds globally minimal solutions, i.e., the D, G, and $H$ instances [Adams, Balas \& Zawack, 1988], TA can compete with SA1. For the remaining, more interesting, instances, TA is clearly outperformed by SA1. The difference becomes again quite pronounced for the largest instances. For the I instances, SA1 finds makespans that are on the average $4.2 \%$ shorter than those found by $\mathrm{TA}$.

(iv) The performance of SA1 and GLS2 is about equal, although for the largest instances SA1 consistently finds slightly better makespans. For the $\mathrm{C}$ instances, for example, SA1 is on the average $1.6 \%$ better and for the I instances $1.3 \%$.

\subsection{When time is of no concern}

It is often argued that randomized variants of local search, such as simulated annealing and genetic local search, show their real strength when running times are of no concern. To verify this, we ran the algorithms SA1 and GLS2 for the ten tough instances identified by Applegate \& Cook [1991]. Three of these instances are due to Adams, Balas \& Zawack [1988]; the others are due to Lawrence [1984] and belong to the 43 instances considered in the previous section. For each instance, SA1 was run with parameter values $\chi_{0}=0.95, \delta=10^{-4}$, and $\varepsilon_{s}=10^{-5}$; a single run took between two and 15 hours. GLS2 was given the same amount of time by tuning the population size. Table 4

\begin{tabular}{lcrrrrrrl}
\hline Instance & $\mathbf{n}$ & $\mathbf{m}$ & \multicolumn{7}{c}{$f$} & LB & status \\
\cline { 3 - 7 } & & & SA1 & GLS2 & A\&C & B\&C & & \\
\hline ABZ7 & 20 & 15 & $668^{*}$ & 680 & $668^{*}$ & 680 & 654 & open \\
ABZ8 & 20 & 15 & $670^{*}$ & 698 & 687 & 701 & 635 & open \\
ABZ9 & 20 & 15 & $691^{*}$ & 708 & 707 & 717 & 656 & open \\
B1 & 15 & 10 & $1053^{*}$ & 1055 & $1053^{*}$ & 1071 & 1040 & open \\
B4 & 15 & 10 & $935^{*}$ & 938 & $935^{*}$ & 953 & 935 & solved \\
B5 & 15 & 10 & 983 & 985 & $977^{*}$ & 988 & 977 & solved \\
C2 & 20 & 10 & $1249^{*}$ & 1265 & 1269 & 1256 & 1235 & open \\
C4 & 20 & 10 & $1185^{*}$ & 1217 & 1195 & 1205 & 1120 & open \\
I3 & 15 & 15 & $1208^{*}$ & 1248 & 1209 & 1226 & 1184 & open \\
I5 & 15 & 15 & 1225 & 1233 & $1222^{*}$ & 1246 & 1222 & solved \\
\hline
\end{tabular}

Table 4. Average makespan $\bar{f}$ when time is of no concern for simulated annealing (SA1), and genetic local search (GLS2) with the $N_{1}$ and $N_{2}$ neighborhoods, respectively, the shuffle algorithm of Applegate \& Cook ( $\& \& C)$, the tabu search algorithm of Barnes \& Chambers (B\&C), the lower bounds from Applegate \& Cook (LB), and the current status; a "*" indicates the best known result.

shows the results we obtained. For purposes of reference the table also includes the values found by Applegate \& Cook's [1991] shuffle algorithm and by Barnes \& Chambers' [1991] implementation of tabu search, as well as the lower bounds on the optimum found by Applegate \& Cook's [1991] edge 
finder algorithm. Edge finder is a branch and bound algorithm, inspired by the work of Carlier \& Pinson [1984], and shuffle is a heuristic variant of edge finder. For details we refer to the paper by Applegate \& Cook.

It appears that, when time is of no concern, substantially improved results are obtained; c.f. Table 3. Furthermore, SA1 outperforms GLS2 even more clearly than before. SA1 also obtains generally better solutions than the approximation algorithms of Applegate \& Cook and Barnes \& Chambers, especially for the open problems. It should be taken into account, however, that their methods are much faster, but also much more tailored towards the specific structure of the job shop scheduling problem. In any case, SA1 has found better solutions than were previously known for five of the ten tough instances.

\section{Discussion}

We have compared a number of local search approaches by applying them to the job shop scheduling problem. From the computational results it can be concluded that the effectiveness of a standard iterative improvement algorithm can be improved either by relaxing the rigid concept of accepting cost improving solutions only, leading to threshold accepting and simulated annealing, or by using concepts from population genetics. This conclusion is in accordance with those drawn by Ulder et al. [1990] from a similar comparison for the traveling salesman problem, with the small but intriguing difference that, for job shop scheduling, threshold accepting is clearly outperformed by both simulated annealing and genetic local search, whereas for the traveling salesman problem threshold accepting and simulated annealing are about equally effective.

Finally, our experiments confirm that simulated annealing, when it is given enough time, can find better solutions than a number of faster and more tailored heuristics.

\section{Bibliography}

Aarts, E.H.L., J.H.M. Korst, P.J. Zwietering [1992], Deterministic and Randomized Local Search, Manuscript NR NL-M.S.17.235, Philips Research Laboratories, Eindhoven, The Netherlands.

AARTS, E.H.L., J.H.M. Korst [1989], Simulated Annealing and Boltzmann Machines, Wiley, Chichester.

ADAMS, J., E. BALAS, D. ZAWACK [1988], The shifting bottleneck procedure for job shop scheduling, Management Science 34, 391-401.

Applegate, D., W. CooK [1991], A computational study of the job-shop scheduling prolilem, ORSA Journal on Computing 3, 149-156.

Barnes, J.W., J.B. Chambers [1991.], Solving the Job Shop Scheduling Problem Using Tabu Search, Technical Report ORP91-06, Graduate Program in Operations Research, University of Texas, Austin, USA.

BAUM, E.B. [1986], Towards practical "neural" computation for combinatorial optimization problems, in: J.S. Denker (ed.) Neural Networks for Computing, AIP Conf. Proc. 151, Snowbird, UT, USA, 53-58.

CARLIER, J., E. PINSON [1989], An algorithm for solving the job shop problem, Management Science 35, 164-176.

DUECK, G., [1990], private communication.

DUECK, G., T. SCHEUER [1990], Threshold accepting: a general purpose optimization algorithm, Journal 
of Computational Physics 90, 161-175.

Fischer, H., G.L. THOMPSON [1963], Probabilistic learning combinations of local job-shop scheduling rules, in: J.F. Muth, G.L. Thompson, eds., Industrial Scheduling, Prentice Hall, Englewood Cliffs, N.J., 225-251.

FRENCH, S. [1982|, Sequencing and Scheduling: An Introduction to the Mathematics of the Job-Shop, Horwood, Chichester.

GAREY, M.R., D.S. Johnson [1979], Computers and Intractability: a Guide to the Theory of NP. completeness, Freeman and Company, San Francisco.

Glover, F. [1989], Tabu search - Part I, ORSA Journal on Computing 1, 190-206.

Glover, F. [1989], Tabu search - Part II, ORSA Journal on Computing 2, 4-32.

GOLDBERG, D.E. [1989], Genetic Algorithms in Search, Optimization and Machines Learning, AddisonWesley, Reading (MA).

JoG, P., J.Y. SUH, D. VAN GUCHT [1989], The effects of population size, heuristic crossover and local improvement on a genetic algorithm for the traveling salesman problem, Proc. Srd Int. Conference on Genetic Algorithms, 110-115.

Johnson, D.S., C.R. ARAGon, L.A. MCGeOCH, C. SChevon [1989], Optimization by simulated annealing: an experimental evaluation; Part I, graph partitioning, Operations Research 37, 865-892.

Johnson, D.S., C.R. ARAGON, L.A. MCGEOCH, C. SChEvon [1991], Optimization by simulated annealing: an experimental evaluation; Part II, graph coloring and number partitioning, Operations Research 39, 378-406. manuscript, Bell Laboratories, Murray Hill, USA.

KirkPatrick, S., C.D. GelatT JR., M.P. VeCCHI [1983], Optimization by simulated annealing, Science 220, 671-680.

LAARHoven, P.J.M. VAN, E.H.L. AARTS [1987], Simulated Annealing: Theory and Applications, Reidel, Dordrecht.

LaARhoven, P.J.M. VAN, E.H.L. Aarts, J.K. Lenstra [1988], Job shop scheduling by simulated annealing, Operations Research 40, 113-126.

Lawler, E.L., J.K. Lenstra, A.H.G. Rinnooy Kan, D.B. Shmoys, eds. [1985], The Traveling Salesman Problem: A Guided Tour of Combinatorial Optimization, Wiley, Chichester.

LAWler, E.L., J.K. Lenstra, A.H.G. Rinnooy KAN, D.B. ShMoYs [1989], Sequencing and Scheduling: Algorithms and Complexity, Designing Decision Support Systems Notes, NFI 11.89/63, Eindhoven University of Technology, Eindhoven, The Netherlands.

Matsuo, H., C.J. SUH, R.S. Sullivan [1988], A controlled search simulated annealing method for the general job shop scheduling problem, Working Paper 03-04-88, Graduate School of Business, The University of Texas at Austin, Austin, USA.

MÜHLENBEIN, H., M. GORGES-SCHLEUTER, O. KRÄMER [1988], Evolution algorithms in combinatorial optimization, Parallel Computing 7, 65-85.

PaPadimitriou, C.H., K. Steiglitz [1982], Combinatorial Optimization: Algorithms and Complexity, Prentice-Hall, Englewood Cliffs, N.J. USA.

ROY, B., B. SUSSMANN [1964], Les problemes d'ordonnancement avec constraints disjonctives, Note DS No. 9 bis, SEMA, Paris, France.

Ulder, N.L.J. [1990], Genetic Local Search: A Population-based Search Algorithm, Technical Note NR 050/90, Philips Research Laboratories, Eindhoven, The Netherlands.

Ulder, N.L.J., E.H.L. Aarts, H-J. Bandelt, P.J.M. van LaArhoven, E. Pesch [1990], Genetic local search for the traveling salesman problem, Lecture Notes in Computer Science 496, Springer, Berlin, 109-116.

YaNNAKAKIS, M. [1990], The analysis of local search problems and their heuristics, Lecture Notes in Computer Science $\mathbf{4 1 5}$, Springer, Berlin, 298-310. 
List of COSOR-memoranda - 1992

\begin{tabular}{|c|c|c|c|}
\hline Number & Month & Author & Title \\
\hline $92-01$ & January & F.W. Steutel & On the addition of log-convex functions and sequences \\
\hline $92-02$ & January & P. v.d. Laan & Selection constants for Uniform populations \\
\hline $92-03$ & February & $\begin{array}{l}\text { E.E.M. v. Berkum } \\
\text { H.N. Linssen } \\
\text { D.A. Overdijk }\end{array}$ & Data reduction in statistical inference \\
\hline $92-04$ & February & $\begin{array}{l}\text { H.J.C. Huijberts } \\
\text { H. Nijmeijer }\end{array}$ & $\begin{array}{l}\text { Strong dynamic input-output decoupling: } \\
\text { from linearity to nonlinearity }\end{array}$ \\
\hline $92-05$ & March & $\begin{array}{l}\text { S.J.L. v. Eijndhoven } \\
\text { J.M. Soethoudt }\end{array}$ & $\begin{array}{l}\text { Introduction to a behavioral approach } \\
\text { of continuous-time systems }\end{array}$ \\
\hline $92-06$ & April & $\begin{array}{l}\text { P.J. Zwietering } \\
\text { E.H.L. Aarts } \\
\text { J. Wessels }\end{array}$ & The minimal number of layers of a perceptron that sorts \\
\hline $92-07$ & April & F.P.A. Coolen & $\begin{array}{l}\text { Maximum Imprecision Related to Intervals of Measures } \\
\text { and Bayesian Inference with Conjugate Imprecise Prior } \\
\text { Densities }\end{array}$ \\
\hline $92-08$ & May & $\begin{array}{l}\text { I.J.B.F. Adan } \\
\text { J. Wessels } \\
\text { W.H.M. Zijm }\end{array}$ & $\begin{array}{l}\text { A Note on "The effect of varying routing probability in } \\
\text { two parallel queues with dynamic routing under a } \\
\text { threshold-type scheduling" }\end{array}$ \\
\hline $92-09$ & May & $\begin{array}{l}\text { I.J.B.F. Adan } \\
\text { G.J.J.A.N. v. Houtum } \\
\text { J.v.d. Wal }\end{array}$ & $\begin{array}{l}\text { Upper and lower bounds for the waiting time in the } \\
\text { symmetric shortest queue system }\end{array}$ \\
\hline $92-10$ & May & P. v.d. Laan & Subset Selection: Robustness and Imprecise Selection \\
\hline $92-11$ & May & $\begin{array}{l}\text { R.J.M. Vaessens } \\
\text { E.H.L. Aarts } \\
\text { J.K. Lenstra }\end{array}$ & $\begin{array}{l}\text { A Local Search Template } \\
\text { (Extended Abstract) }\end{array}$ \\
\hline $92-12$ & May & F.P.A. Coolen & $\begin{array}{l}\text { Elicitation of Expert Knowledge and Assessment of Im- } \\
\text { precise Prior Densities for Lifetime Distributions }\end{array}$ \\
\hline $92-13$ & May & $\begin{array}{l}\text { M.A. Peters } \\
\text { A.A. Stoorvogel }\end{array}$ & Mixed $H_{2} / H_{\infty}$ Control in a Stochastic Framework \\
\hline
\end{tabular}




\begin{tabular}{llll} 
Number & Month & Author & Title \\
\hline $92-14$ & June & P.J. Zwietering & The construction of minimal multi-layered perceptrons: \\
& & E.H.L. Aarts & a case study for sorting \\
& J. Wessels &
\end{tabular}

92-15 June P. van der Laan

Experiments: Design, Parametric and Nonparametric Analysis, and Selection

92-16 June J.J.A.M. Brands On the number of maxima in a discrete sample

F.W. Steutel

R.J.G. Wilms

92-17 June S.J.L. v. Eijndhoven Introduction to a behavioral approach of continuous-time J.M. Soethoudt systems part II

92-18 June J.A. Hoogeveen New lower and upper bounds for scheduling around a H. Oosterhout small common due date

S.L. van der Velde

92-19 June F.P.A. Coolen

On Bernoulli Experiments with Imprecise Prior Probabilities

92-20 June J.A. Hoogeveen Minimizing Total Inventory Cost on a Single Machine

S.L. van de Velde in Just-in-Time Manufacturing

92-21 June J.A. Hoogeveen Polynomial-time algorithms for single-machine

S.L. van de Velde bicriteria scheduling

92-22 June P. van der Laan The best variety or an almost best one? A comparison of subset selection procedures

92-23 June T.J.A. Storcken Extensions of choice behaviour

P.H.M. Ruys

92-24 July L.C.G.J.M. Habets Characteristic Sets in Commutative Algebra: an overview

92-25 July P.J. Zwietering Exact Classification With Two-Layered Perceptrons

E.H.L. Aarts

J. Wessels

92-26 July M.W.P. Savelsbergh Preprocessing and Probing Techniques for Mixed Integer Programming Problems 


\begin{tabular}{llll} 
Number & Month & Author & Title \\
\hline $92-27$ & July & I.J.B.F. Adan & Analysing $E_{k}\left|E_{r}\right| c$ Queues \\
& & W.A. van de \\
& & Waarsenburg & \\
& J. Wessels &
\end{tabular}

92-28 July O.J. Boxma

The compensation approach applied to a $2 \times 2$ switch

G.J. van Houtum

92-29 July E.H.L. Aarts Job Shop Scheduling by Local Search

P.J.M. van Laarhoven

J.K. Lenstra

N.L.J. Ulder 